\title{
Caractérisation de champignons polycentriques du rumen observés in vivo
}

\author{
A Breton ${ }^{1,3}, M_{\text {Dusser }}^{2}$, B Gaillard-Martinie ${ }^{3}$, \\ $\mathrm{J}_{\text {Guillot }}{ }^{2}$, L Millet $^{3}$ \\ 1 Université Clermont II, laboratoire de biologie comparée des protistes, \\ 63170 Aubières; \\ 2 Université Clermont I, faculté de pharmacie, laboratoire de microbiologie, \\ place H-Dunant, 63000, Clermont-Ferrand; \\ 3 INRA, centre de Clermont-Ferrand-Theix, laboratoire de microbiologie, \\ 63122 Saint-Genès-Champanelle, France
}

Summary - Characterization of rumen polycentric fungi observed in vivo. Neocallimastix joyonii and Anaeromyces mucronatus were characterised in vivo. The morphological features and the cell wall composition of the sporocysts were similar to those observed in culture.

Les champignons anaérobies stricts du rumen des genres Neocallimastix, Piromonas ut Sphaeromonas ont tous été jusqu'ici caractérisés en culture pure selon des critères morphologiques, métaboliques (Breton et al, 1989) et cytologiques (Gaillard et al, 1989; Guillot et al, 1990). Dans le rumen, des champignons ont été observés fixés aux substrats végétaux, mais sans être rapportés aux espèces décrites en culture in vitro (Grenet et al, 1989).

L'objet de cette note est de décrire 2 organismes polycentriques, Neocallimastix joyonii (Breton et al, 1989) et Anaeromyces mucronatus (Breton et al, 1990 ), obtenus in vivo, et de comparer les observations avec celles réalisées en culture pure.

Matériel et méthodes - Des téguments de graines de soja placés dans des sacs en nylon ont été introduits dans le rumen de vaches fistulisées. A la suite d'isolements en milieu gélosé, il avait été montré que ces rumens étaient colonisés par $N$ joyonii et $A$ mucronatus à l'exclusion de toute autre espèce fongique. Après $48 \mathrm{~h}$ de séjour dans le rumen, les téguments des graines avec les champignons fixés ont été prélevés d'une part pour être traités de façon classique pour l'observation en microscopie électronique à balayage (Breton et al, 1989), d'autre part en vue d'une étude des parois fongiques à l'aide de lectines marquées à la fluorescéine spécifiques du L-fucose (lectines extraites de Laccaria amethystina, Ulex europaeus, Pholiota squarrosa, Clitocybe geotropa), du D-mannose et du D-glucose (Lectine de Lens culinaris), de la $N$ acetyl-galactosamine (lectine de Glycine max) et du diacetyl chitobiose (lectine de Datura stramonium) (Guillot et al, 1990). Après 3 lavages dans du tampon phospate, $0,01 \mathrm{M}$, pH 7,2, les échantillons prélevés ont été mis au contact de la lectine à tester, à $4^{\circ} \mathrm{C}$, pendant $30 \mathrm{~min}$, puis rincés et montés entre lame et lamelle dans du tampon phosphate, pour être observés en microscopie de fluorescence.

Résultats et discussion $-N$ joyonii et $A$ mucronatus fructifient au niveau de l'assise palissadique et du hile des téguments de soja. En l'absence de tout rhizomycélium visible, il est impossible de conclure, dans ces conditions, à la nature polycentrique de ces espèces.

$N$ joyonii différencie sur les téguments de soja des sporocystes souvent grégaires, sub-globuleux, $49(60) 71 \times$ 49(56) $71 \mu \mathrm{m}$, sessiles ou courtement pédicellés (fig 1). Une très forte réactivité aux lectines de Laccaria amethystina, Ulex europaeus, Pholiota squarrosa et Clitocybe geotropa témoigne de la présence de fucose dans la paroi des sporocystes et de leur pédon- 


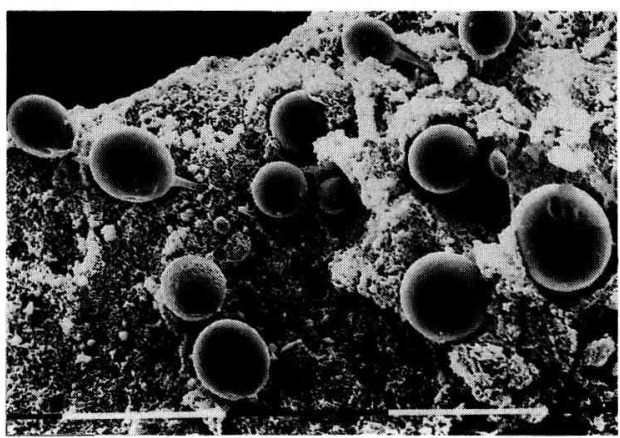

Fig 1. Neocallimastix joyonii sporocystes en MEB $\times 140$.

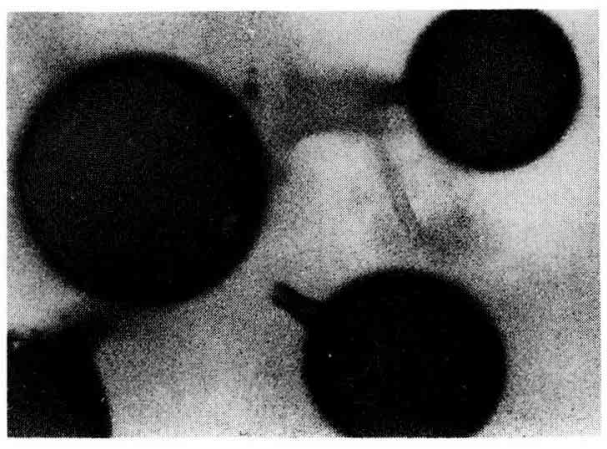

Fig 2. Neocallimastix joyonii sporocystes en fluorescence avec la lectine de $C$ geotropa $\times 420$.

cule (fig 2), les autres lectines donnant des résultats négatifs.

A mucronatus forme in vivo des sporocystes isolés ou peu groupés, ellipsoïdaux, $52(62) 72 \times 18(24) 32 \mu \mathrm{m}$, mucronés au sommet et portés par un pédicelle qui émerge toujours nettement du substrat (figs 3 et 4 ). II se caractérise en outre par l'absence de toute réactivité des structures reproductrices aux différentes lectines testées.

En conclusion, $N$ joyonii et $A$ mucronatus différencient dans le rumen des sporocystes morphologiquement et cytologiquement très différents d'une espèce à l'autre et conformes à ceux

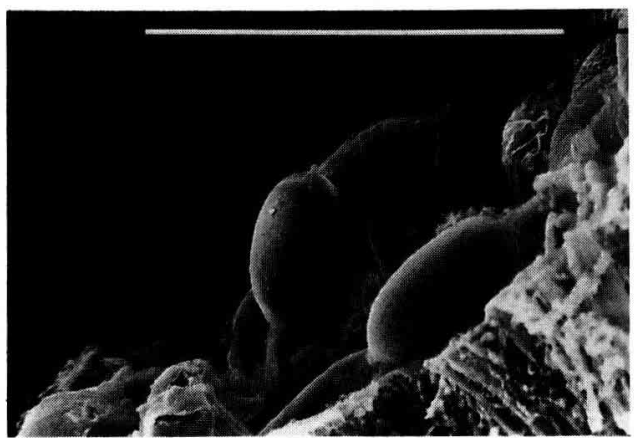

Fig 3. Anaeromyces mucronatus sporocystes en MEB $\times 350$.

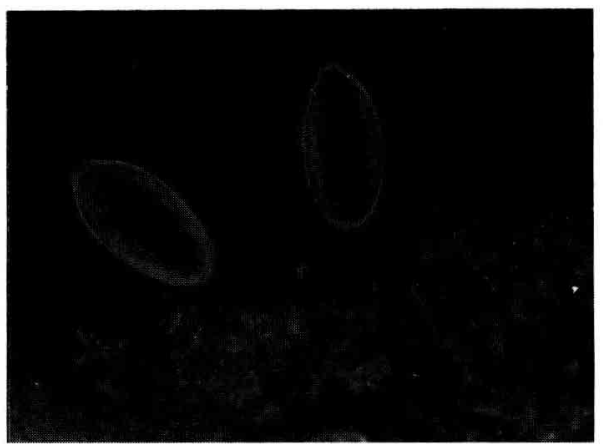

Fig 4. Anaeromyces mucronatus sporocystes en microscopie photonique $\times 330$.

décrits en culture pure (Breton et al, $1989,1990)$. En conséquence, ces deux espèces sont identifiables in vivo.

Breton A, Bernalier A, Bonnemoy F, Fonty G, Gaillard G, Gouet $P$ (1989) FEMS Microbiol Lett 58, 309-314

Breton A, Bernalier A, Dusser M, Fonty G, Gaillard-Martinie B, Guillot J (1990) FEMS Microbiol Lett 58, 309-314

Gaillard B, Breton A, Bernalier A (1989) Curr Microbiol, 19, 103-107

Grenet E, Breton A, Barry P, Fonty G (1989) Anim Feed Sci Technol 26, 55-70

Guillot J, Breton A, Damez $M$, Dusser $M$, Gaillard-Martinie B, Millet L (1990) FEMS Microbiol Lett 67, 151-156 